\title{
A Generic Algebraic Proof of the Unified Power Conservative Equivalent Circuit Theorem
}

\author{
Ivo Barbi, Life Fellow, IEEE
}

\begin{abstract}
This paper presents a generic algebraic proof of a recently published theorem [4], on the power conservative equivalent circuit for linear DC networks formed by timeinvariant resistors and independent voltage and current sources. As the cited publication states, the internal losses of any network have two components: one variable and dependent on the internal resistances of the actual circuit and the power transferred to the pair of accessible terminals; and the other constant and dependent only on the internal voltage and current sources and the resistances of the actual network. It is also noted that the traditional Thévenin and Norton equivalent circuits are particular cases of the proposed equivalent circuit.
\end{abstract}

Index Terms-DC networks, Thévenin, Norton, power ponservative, DC network theorem, equivalent circuit.

\section{INTRODUCTION}

$\mathbf{T}$ HE Thevenin [1] and Norton [2] equivalent circuits are important to the theory of DC networks and are commonly used in the analysis of electrical circuits, since they provide simplicity in many practical applications.

However, it is well known that these approaches are limited to representing the phenomena that occur in the actual circuit, since they can only be used to determine voltage and current at a pair of accessible terminals of the network. Therefore, these traditional equivalent circuits are not appropriate for determining the efficiency and power of the actual network.

In [3-4] it is demonstrated, using simplified analysis and case studies, that any linear and bilateral DC network formed by time-invariant resistors and independent voltage and current sources, with a pair of accessible terminals $a b$, can be replaced by the equivalent circuit shown in Fig. 1. In this paper a generic algebraic proof of the proposed theorem is presented.

\section{Statement Of The Unified Power Conservative EQUiVALENT CIRCUIT THEOREM}

As stated in [4], Theorem: Any linear DC network consisting of independent voltage and current sources, and resistors, with two accessible terminals (Fig. 1(a)) can be replaced by an equivalent circuit with a DC voltage source $V_{T 1}$, a DC current source $I_{N 1}$ and three resistors $R_{X}, R_{y}$ and $R_{T}$ (Fig. 1(b)). The voltage $V_{T 1}$ is measured at the open terminals, with all internal current sources of the network set equal to zero. The current $I_{N 1}$ is the current through a short circuit applied to the external terminals, with all network internal voltage sources set equal to zero. The resistance $R_{X}$ associated in parallel with the voltage source $V_{T 1}$ is given by the equation $R_{X}=\frac{V_{T 1}{ }^{2}}{P_{X}}$, where $P_{X}$ is the power supplied by the internal

I. Barbi is with the Department of Electrical Engineering, Federal University of Santa Catarina, Florianopolis, SC, Brazil. e-mail: ivobarbi@gmail.com. voltage sources and dissipated internally in the circuit with the external terminals open and all internal current sources set equal to zero. The resistance $R_{y}$ associated in series with the current source $I_{N 1}$ is given by $R_{y}=\frac{P_{y}}{I_{N 1}{ }^{2}}$, where $P_{y}$ is the power supplied by the internal current sources and dissipated in the network, with a short circuit applied to the external terminals and all internal voltage sources set equal to zero. The resistance $R_{T}$ is that measured at the terminals with all internal voltage replaced by a short circuit and all internal current sources replaced by an open circuit.

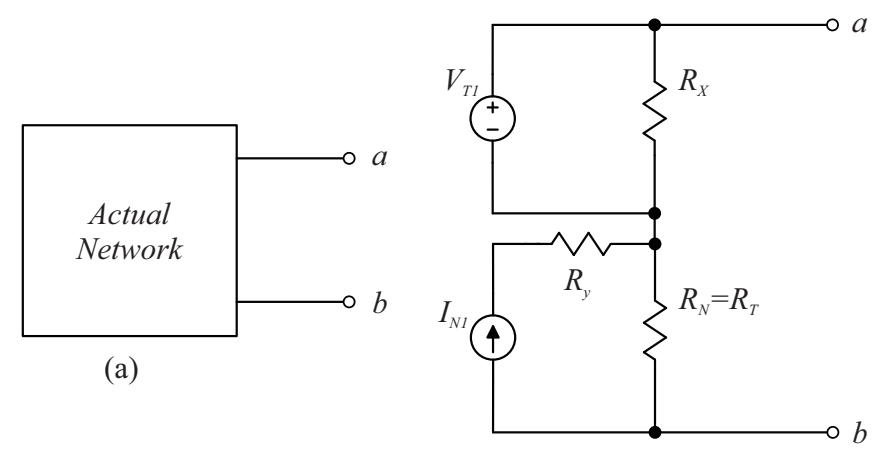

(b)

Fig. 1. The power conservative unified equivalent circuit of DC networks consisting of independent voltage and current sources, and resistors.

\section{Generic Algebraic Proof Of The Theorem}

Fig. 2 shows a reciprocal and symmetric DC network formed by two independent ideal voltage sources $V_{1}$ and $V_{2}$, two independent ideal current sources $I_{1}$ and $I_{2}$, and a pair of accessible terminals ab to which an external voltage source $V_{o}$ is connected. The resistors of the network are in the box denoted by $R$.

The equations that describe the network in terms of inverse hybrid parameters (or g-parameters) [2] are

$$
\begin{aligned}
& I_{1}=g_{11} V_{1}+g_{12} V_{2}+g_{1 p} I_{p}+g_{1 q} I_{q}+g_{1 o} V_{o} \\
& I_{2}=g_{21} V_{1}+g_{22} V_{2}+g_{2 p} I_{p}+g_{2 q} I_{q}+g_{2 o} V_{o} \\
& V_{p}=g_{p 1} V_{1}+g_{p 2} V_{2}+g_{p p} I_{x}+g_{p q} I_{q}+g_{p o} V_{o} \\
& V_{q}=g_{q 1} V_{1}+g_{q 2} V_{2}+g_{q p} I_{p}+g_{q q} I_{q}+g_{q o} V_{o}
\end{aligned}
$$




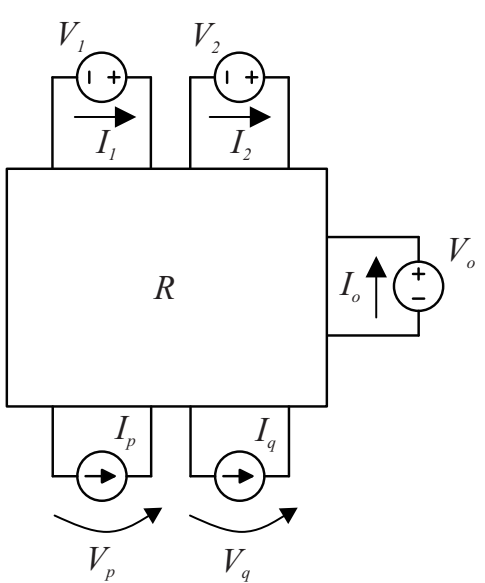

Fig. 2. DC network formed by resistors, independent voltage and current sources, and a pair of accessible terminals $a b$.

$$
I_{o}=g_{o 1} V_{1}+g_{o 2} V_{2}+g_{o p} I_{p}+g_{o q} I_{q}+g_{o o} V_{o}
$$

which may also be written in the matrix form

$$
\left[\begin{array}{c}
I_{1} \\
I_{2} \\
V_{p} \\
V_{q}
\end{array}\right]=\left[\begin{array}{llll}
g_{11} & g_{12} & g_{1 p} & g_{1 q} \\
g_{21} & g_{22} & g_{2 p} & g_{2 q} \\
g_{p 1} & g_{p 2} & g_{p p} & g_{p q} \\
g_{q 1} & g_{q 2} & g_{q p} & g_{q q}
\end{array}\right] \cdot\left[\begin{array}{c}
V_{1} \\
V_{2} \\
I_{p} \\
I_{q}
\end{array}\right]+\left[\begin{array}{c}
g_{1 o} \\
g_{2 o} \\
g_{p o} \\
g_{q o}
\end{array}\right] . V_{o}
$$

$$
I_{o}=\left[\begin{array}{llll}
g_{o 1} & g_{o 2} & g_{o p} & g_{o q}
\end{array}\right] \cdot\left[\begin{array}{c}
V_{1} \\
V_{2} \\
I_{p} \\
I_{q}
\end{array}\right]+g_{o o} V_{o}
$$

For reciprocal and symmetric networks, $g_{21}=g_{12}, g_{p q}=$ $g_{q p}, g_{o 1}=g_{1 o}, g_{o 2}=g_{o 2}, g_{p 1}=-g_{1 p}, g_{p 2}=-g_{2 p}, g_{q 1}=$ $-g_{1 q}, g_{q 2}=-g_{2 q}, g_{p o}=-g_{o p}, g_{q o}=-g_{o q}, g_{p o}=-g_{o p}$ and $g_{q o}=-g_{o q}$.

Let $G$ be defined as

$$
G=\left[\begin{array}{llll}
g_{11} & g_{12} & g_{1 p} & g_{1 q} \\
g_{21} & g_{22} & g_{2 p} & g_{2 q} \\
g_{p 1} & g_{p 2} & g_{p p} & g_{p q} \\
g_{q 1} & g_{q 2} & g_{q p} & g_{q q}
\end{array}\right]
$$

The substitution of (8) in (6) leads to

$$
\left[\begin{array}{c}
I_{1} \\
I_{2} \\
V_{p} \\
V_{q}
\end{array}\right]=G \cdot\left[\begin{array}{c}
V_{1} \\
V_{2} \\
I_{p} \\
I_{q}
\end{array}\right]+\left[\begin{array}{c}
V_{1} \\
V_{2} \\
I_{p} \\
I_{q}
\end{array}\right] \cdot V_{o}
$$

\section{A. The Voltage At Terminals ab When They Are Open $\left(I_{o}=0\right)$}

Let us consider the condition in which the external voltage source is disconnected from the terminals $a b$. The current $I_{o}=$ 0 and the voltage $V_{a b}$ is the voltage of the Thévenin equivalent circuit, denoted by $V_{T}$. The representation of the network for this condition is shown in Fig. 3.

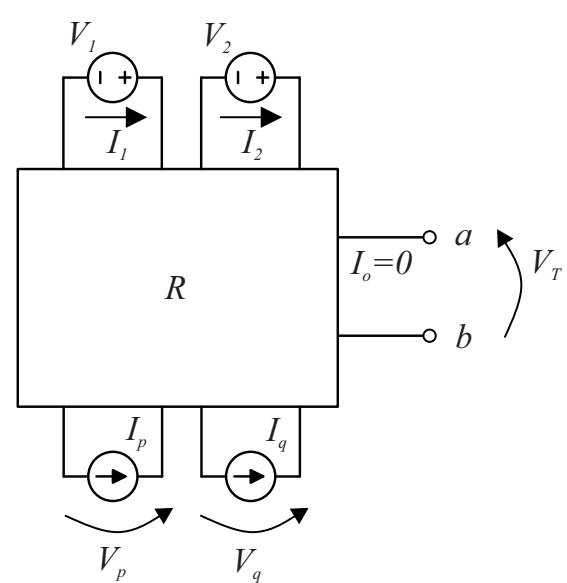

Fig. 3. The network with the terminals $a b$ open.

Making $I_{o}=0$ in (5) we find

$$
0=g_{o 1} V_{1}+g_{o 2} V_{2}+g_{o p} I_{p}+g_{o q} I_{q}+g_{o o} V_{T}
$$

Hence,

$$
V_{T}=-\frac{1}{g_{o o}}\left[\begin{array}{llll}
g_{o 1} & g_{o 2} & g_{o p} & g_{o q}
\end{array}\right]\left[\begin{array}{c}
V_{1} \\
V_{2} \\
I_{p} \\
I_{q}
\end{array}\right]
$$

Let us define $V_{T 1}$ and $V_{T 2}$ as

$$
\begin{aligned}
& V_{T 1}=-\frac{1}{g_{o o}}\left[\begin{array}{ll}
g_{o 1} & g_{o 2}
\end{array}\right]\left[\begin{array}{c}
V_{1} \\
V_{2}
\end{array}\right] \\
& V_{T 2}=-\frac{1}{g_{o o}}\left[\begin{array}{ll}
g_{o p} & g_{o q}
\end{array}\right]\left[\begin{array}{c}
I_{p} \\
I_{q}
\end{array}\right]
\end{aligned}
$$

The substitution of (12) and (13) in (11) leads to

$$
V_{T}=V_{T 1}+V_{T 2}
$$

which is a consequence of the superposition principle. The voltage $V_{T 1}$ is the open voltage at terminals $a b$ when the current sources $I_{p}=I_{q}=0$ while $V_{T 2}$ is the open voltage at the same terminals when the voltage sources $V_{1}=V_{2}=0$, as shown in Fig. 4.

\section{B. Output Current When $V_{1}=V_{2}=V_{o}=0$}

Fig. 5 shows the network when $V_{1}=V_{2}=V_{o}=0$. The current $I_{o}$ is the current source of the Norton equivalent circuit under this constraint, denoted by $I_{N 1}$.

Making $V_{1}=V_{2}=V_{o}=0$ in (7) we find

$$
I_{N 1}=g_{o p} I_{p}+g_{o q} I_{q}
$$

Substitution of (15) in (13) gives

$$
V_{T 2}=-\frac{1}{g_{o o}} I_{N 1}
$$

where $V_{T 2}$ is the voltage at the open terminals $a b$ when $V_{1}=$ $V_{2}=0, I_{p} \neq 0$ and $I_{q} \neq 0$. 


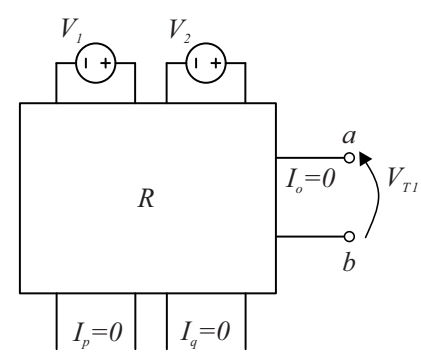

(a)

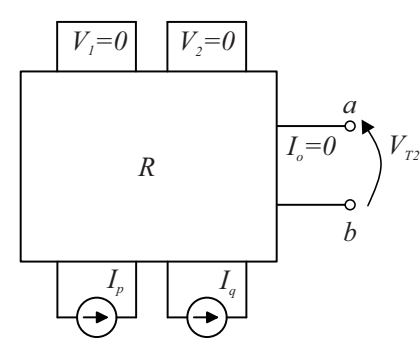

(b)
Fig. 4. The network with the terminals $a b$ open. (a) when $I_{p}=I_{q}=0$ and (b) when $V_{1}=V_{2}=0$.

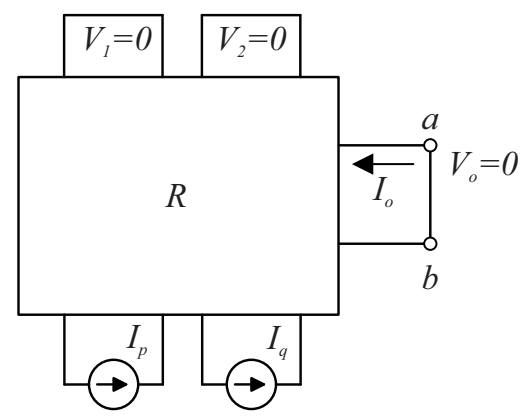

Fig. 5. The network when $V_{1}=V_{2}=V_{o}=0, I_{p} \neq 0$ and $I_{p} \neq 0$.

\section{The Resistance $R_{T}$ of the Thévenin Equivalent Circuit}

The resistance of the Thévenin equivalent circuit is the driving point resistance of the network at the terminals $a b$ when all internal voltage sources are replaced by a short circuit and all internal current sources are replaced by an open circuit, as shown in Fig. 6.

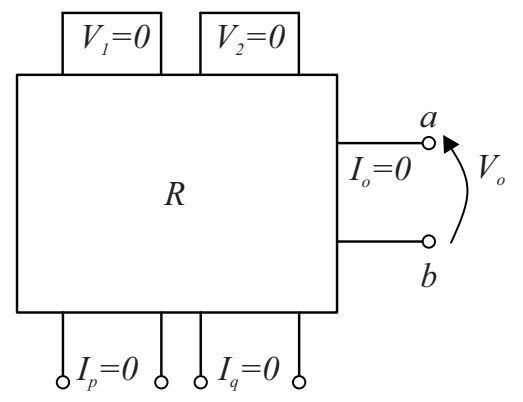

Fig. 6. Measurement of the resistance $R_{T}$ from the terminals $a b$.

Making $V_{1}=V_{2}=I_{p}=I_{q}=0$ in expression (7) we find

$$
R_{T}=\frac{V_{o}}{I_{o}}=-\frac{1}{g_{o o}}
$$

D. Internal Power Dissipated in the Resistors of the Network

The power dissipated by the network resistors is

$$
P=\left[\begin{array}{llll}
V_{1} & V_{2} & I_{p} & I_{q}
\end{array}\right] G\left[\begin{array}{c}
I_{1} \\
I_{2} \\
V_{p} \\
V_{q}
\end{array}\right]+V_{o} I_{o}
$$

Substituting (9) in (18) we find

$$
P=\left[\begin{array}{llll}
V_{1} & V_{2} & I_{p} & I_{q}
\end{array}\right] G\left(\begin{array}{c}
V_{1} \\
V_{2} \\
I_{p} \\
I_{q}
\end{array}\right)+\left(\begin{array}{c}
g_{1 o} \\
g_{2 o} \\
g_{p o} \\
g_{q o}
\end{array}\right) V_{o}+V_{o} I_{o}
$$

Since

$$
\begin{aligned}
& {\left[\begin{array}{llll}
V_{1} & V_{2} & I_{p} & I_{q}
\end{array}\right] G\left[\begin{array}{l}
I_{1} \\
I_{2} \\
V_{p} \\
V_{q}
\end{array}\right]=\left[\begin{array}{ll}
V_{1} & V_{2}
\end{array}\right]\left[\begin{array}{ll}
g_{11} & g_{12} \\
g_{12} & g_{22}
\end{array}\right]\left[\begin{array}{l}
V_{1} \\
V_{2}
\end{array}\right]} \\
& +\left[\begin{array}{ll}
I_{p} & I_{q}
\end{array}\right]\left[\begin{array}{ll}
g_{p p} & g_{p q} \\
g_{p q} & g_{q q}
\end{array}\right]\left[\begin{array}{l}
I_{p} \\
I_{q}
\end{array}\right]
\end{aligned}
$$

the expression (19) becomes

$$
\begin{aligned}
& P=\left[\begin{array}{ll}
V_{1} & V_{2}
\end{array}\right]\left[\begin{array}{ll}
g_{11} & g_{12} \\
g_{12} & g_{22}
\end{array}\right]\left[\begin{array}{l}
V_{1} \\
V_{2}
\end{array}\right] \\
& +\left[\begin{array}{ll}
I_{p} & I_{q}
\end{array}\right]\left[\begin{array}{ll}
g_{p p} & g_{p q} \\
g_{p q} & g_{q q}
\end{array}\right]\left[\begin{array}{l}
I_{p} \\
I_{q}
\end{array}\right] \\
& +\left[\begin{array}{llll}
V_{1} & V_{2} & I_{p} & I_{q}
\end{array}\right]\left[\begin{array}{l}
g_{1 o} \\
g_{2 o} \\
g_{p o} \\
g_{q o}
\end{array}\right] V_{o}+V_{o} I_{o}
\end{aligned}
$$

Let us define $P_{a}$ and $P_{b}$ as

$$
\begin{gathered}
P_{a}=\left[\begin{array}{ll}
V_{1} & V_{2}
\end{array}\right]\left[\begin{array}{ll}
g_{11} & g_{12} \\
g_{12} & g_{22}
\end{array}\right]\left[\begin{array}{l}
V_{1} \\
V_{2}
\end{array}\right] \\
P_{b}=\left[\begin{array}{ll}
I_{p} & I_{q}
\end{array}\right]\left[\begin{array}{ll}
g_{p p} & g_{p q} \\
g_{p q} & g_{q q}
\end{array}\right]\left[\begin{array}{c}
I_{p} \\
I_{q}
\end{array}\right]
\end{gathered}
$$

Substitution of (22) and (23) in (21) gives

$P=P_{a}+P_{b}+\left[\begin{array}{llll}V_{1} & V_{2} & I_{p} & I_{q}\end{array}\right]\left[\begin{array}{c}g_{1 o} \\ g_{2 o} \\ g_{p o} \\ g_{q o}\end{array}\right] V_{o}+V_{o} I_{o}$

Substitution of (7) in (24) yields

$P=P_{a}+P_{b}+\left[\begin{array}{llll}V_{1} & V_{2} & I_{p} & I_{q}\end{array}\right]\left[\begin{array}{c}g_{1 o} \\ g_{2 o} \\ g_{p o} \\ g_{q o}\end{array}\right] V_{o}$
$+\left[\begin{array}{llll}g_{1 o} & g_{2 o} & -g_{p o} & -g_{q o}\end{array}\right]\left[\begin{array}{c}V_{1} \\ V_{2} \\ I_{p} \\ I_{q}\end{array}\right]^{V_{o}+g_{o o} V_{o}^{2}}$

We can observe that 


$$
\begin{aligned}
& {\left[\begin{array}{llll}
V_{1} & V_{2} & I_{p} & I_{q}
\end{array}\right]\left[\begin{array}{l}
g_{1 o} \\
g_{2 o} \\
g_{p o} \\
g_{q o}
\end{array}\right] V_{o}} \\
& +\left[\begin{array}{llll}
g_{1 o} & g_{2 o} & -g_{p o} & -g_{q o}
\end{array}\right]\left[\begin{array}{c}
V_{1} \\
V_{2} \\
I_{p} \\
I_{q}
\end{array}\right] \\
& =2\left[\begin{array}{ll}
g_{1 o} V_{1} & g_{2 o} V_{2}
\end{array}\right]
\end{aligned}
$$

and that

$$
g_{1 o} V_{1}+g_{2 o} V_{2}=-g_{o o} V_{T 1}
$$

By substituting (26) and (27) in (25) we obtain

$$
P=P_{a}+P_{b}-2 g_{o o} V_{T 1} V_{o}+g_{o o} V_{o}^{2}
$$

which represents the total power delivered to the resistors of the network by the voltage sources $V_{1}, V_{2}$ and $V_{o}$, and by the current sources $I_{p}$ and $I_{q}$. Expression (28) may also be written in the form

$P=P_{a}+P_{b}-2 g_{o o} V_{T 1} V_{o}+g_{o o} V_{o}^{2}+g_{o o} V_{T 1}^{2}-g_{o o} V_{T 1}^{2}$

Since

$$
g_{o o} V_{o}^{2}-2 g_{o o} V_{T 1} V_{o}+g_{o o} V_{T 1}^{2}=g_{o o}\left(V_{o}-V_{T 1}\right)^{2}
$$

we can write

$$
P=P_{a}-g_{o o} V_{T 1}^{2}+P_{b}+g_{o o}\left(V_{o}-V_{T 1}\right)^{2}
$$

\section{E. Internal Power Dissipated In The Resistors Of The Network} When $I_{p}=I_{q}=I_{o}=0$

Fig. 7 shows the network for the condition where $I_{p}=I_{q}=$ $I_{o}=0, V_{1} \neq 0$ and $V_{2} \neq 0$.

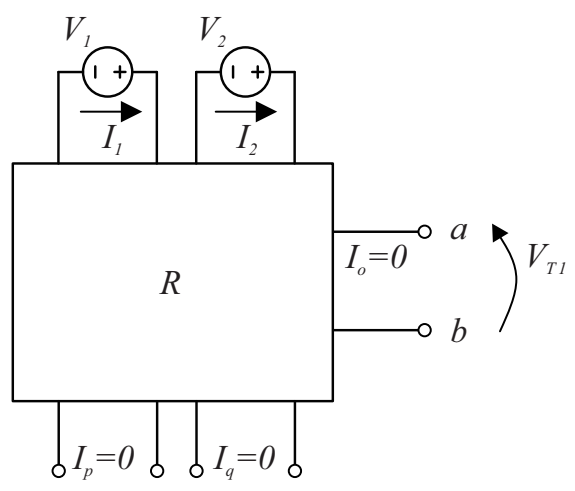

Fig. 7. The network when $I_{p}=I_{q}=I_{o}=0, V_{1} \neq 0$ and $V_{2} \neq 0$.

The voltage at the terminals $a b$ is equal to $V_{T 1}$ and is given by expression (12). Substituting (12) in (29) and making $P_{b}=$ 0 and $V_{O}=V_{T 1}$ we find the power dissipated by the internal resistors of the network when $I_{p}=I_{q}=I_{o}=0$ denoted, by $P_{x}$

$$
P_{x}=P_{a}-g_{o o} V_{T 1}^{2}
$$

Substituting (22) in (32) we find

$$
P_{x}=\left[\begin{array}{ll}
V_{1} & V_{2}
\end{array}\right]\left[\begin{array}{ll}
g_{11} & g_{12} \\
g_{12} & g_{22}
\end{array}\right]\left[\begin{array}{l}
V_{1} \\
V_{2}
\end{array}\right]-g_{o o} V_{T 1}^{2}
$$

From (12) and (33) the equivalent circuit with the resistor $R_{x}$ shown in Fig. 8 is obtained, in which the power $P_{x}$ is dissipated.

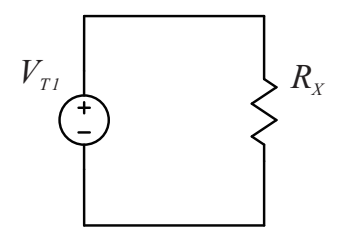

Fig. 8. The network equivalent circuit when $I_{p}=I_{q}=I_{o}=0, V_{1} \neq 0$ and $V_{2} \neq 0$.

The resistance $R_{x}$ is determined by the expression

$$
R_{x}=\frac{V_{T 1}^{2}}{P_{x}}
$$

\section{F. Internal Power Dissipated In The Resistors Of The Network When $V_{1}=V_{2}=V_{o}=0$}

Fig. 5 shows the network for the condition where $V_{1}=$ $V_{2}=V_{o}=0, I_{p} \neq 0$ and $I_{q} \neq 0$, which implies that $V_{T 1}=0$ and $P_{a}=0$.

Let us designate by $P_{y}$ the power dissipated internally in the resistors of the network under this constraint.

Hence,

$$
P_{y}=P_{b}
$$

Substitution of (23) in (33) yields

$$
P_{y}=\left[\begin{array}{ll}
I_{p} & I_{q}
\end{array}\right]\left[\begin{array}{ll}
g_{p p} & g_{p q} \\
g_{p q} & g_{q q}
\end{array}\right]\left[\begin{array}{l}
I_{p} \\
I_{q}
\end{array}\right]
$$

The resistor $R_{y}$, in which the power $P_{y}$ is dissipated is given by

$$
R_{y}=\frac{P_{y}}{I_{N 1}^{2}}
$$

The corresponding equivalent circuit is represented in Fig. 9 . 


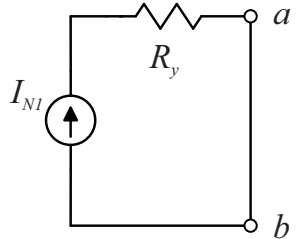

Fig. 9. The equivalent circuit when $V_{1}=V_{2}=V_{o}=0, I_{p} \neq 0$ and $I_{q} \neq 0$.

\section{G. The Unified Equivalent Circuit}

The substitution of (17), (35) and (36) in (31) leads to

$$
P=P_{x}+P_{y}+\frac{\left(V_{o}-V_{T 1}\right)^{2}}{R_{T}}
$$

Since $P_{x}=V_{T 1}{ }^{2} / R_{x}$ and $P_{y}=R_{y} I_{N 1}{ }^{2}$, the expression for the total power dissipated in the internal resistors of the network reduces to the form

$$
P=\frac{V_{T 1}^{2}}{R_{x}}+R_{y} I_{N 1}^{2}+\frac{\left(V_{o}-V_{T 1}\right)^{2}}{R_{T}}
$$

The circuit that satisfies expressions (14), (16) and (39), by writing the Kirchhoff voltage and current laws along with the superposition principle is shown in Fig. 10.

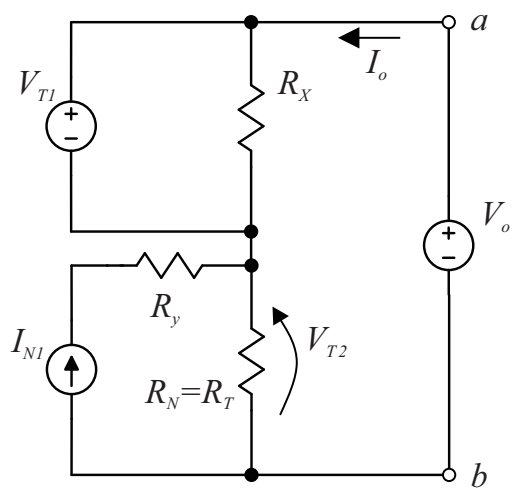

Fig. 10. Power conservative equivalent circuit for the network formed by resistors and independent voltage and current sources.

The losses in $R_{x}$ and $R_{y}$ are not dependent on $V_{o}, I_{o}$ or $P_{o}$. On the other hand, the losses in the resistor $R_{T}$ are dependent on $V_{o}$. Therefore, the internal losses in the network resistors have two components, one of which is dependent on and the other independent of the power measured at terminals $a b$.

The theorem is not restricted to two independent voltage and current sources as shown in Fig. 2. The proof given above can easily be generalized to include any number of voltage and current sources.

\section{Derivation Of ThÉVEnin AND Norton EQUIVALENT CIRCUITS}

Looking at the equivalent circuit shown in Fig. 10, we can conclude that the resistors $R_{x}$ and $R_{y}$, responsible for the constant internal losses in the resistors of the actual network, do not affect the values of $V_{o}, I_{o}$ or $P_{o}$. Therefore, when internal losses can be neglected, these resistors can be excluded from the circuit, resulting in the non-conservative equivalent circuit shown in Fig. 11.

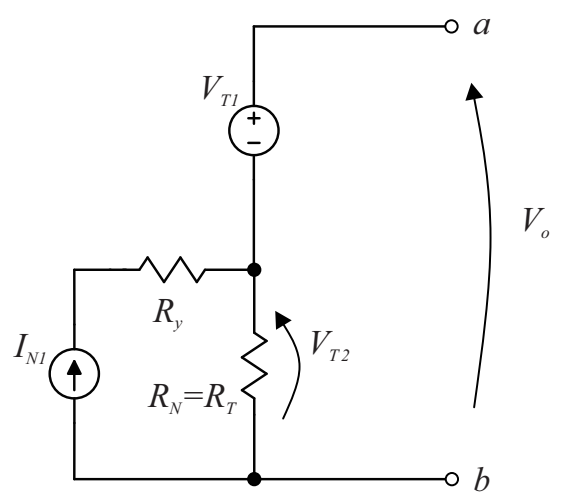

Fig. 11. Non-conservative equivalent circuit for the network formed by resistors and independent voltage and current sources.

The traditional Thévenin equivalent circuit can be derived from Fig. 11, as represented in Fig. 12(a). The equivalent resistance is $R_{T}$ and the voltage source is determined by

$$
V_{T}=V_{T 1}+R_{T} I_{N 1}
$$

The traditional Norton's equivalent circuit can also be derived and is shown in Fig. 12(b), where $R_{N}=R_{T}$ and

$$
I_{N}=I_{N 1}+\frac{V_{T 1}}{R_{N}}
$$

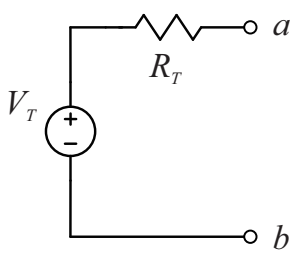

(a)

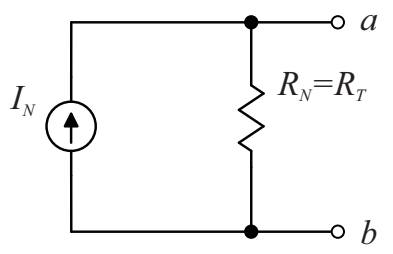

(b)
Fig. 12. Traditional Thévenin equivalent circuit. (b) Traditional Norton equivalent circuit.

\section{CONCLUSION}

The paper has presented a generic algebraic proof of the recently introduced theorem on the unified power conservative equivalent circuit formed by time-invariant resistors, and independent voltage and current sources [3-4]. It is demonstrated that total power is the sum of the powers supplied simultaneously by the voltage sources with all current sources replaced by an open circuit, and the powers supplied by the current sources with all voltage sources replaced by a short circuit. One corollary to the proved theorem is that the internal losses have two components, one being constant and independent of the current or voltage at terminals $a b$, while the other is dependent on this voltage or current. 


\section{ACKNOWLEDGMENT}

The work of Victor Luiz Flor Borges, Engineer with the Brazilian Institute of Power Electronics and Renewable Energy (IBEPE), who collaborated in the manuscript editing, is gratefully acknowledged.

\section{REFERENCES}

[1] Thévenin, "Sur un Nouveaux Théoreme d'Electricité Dynamique", Comptes Rendus des Séances de l'Academie des Sciences, Tome: 97, 1883 , pp. 159-161.

[2] E. L. Norton, "Design of finite networks for uniform frequency characteristics", Bell Laboratories, Tech. Rep, TM26-0-1860, 1926.

[3] I. Barbi, "Power Conservative Equivalent Circuit for DC Networks," in IEEE Access, vol. 8, pp. 113667-113674, 2020, doi: 10.1109/ACCESS.2020.3004026.

[4] I. Barbi, "Unified Power Conservative Equivalent Circuit for DC Networks," in IEEE Access, vol. 8, pp. 178230-178237, 2020, doi: 10.1109/ACCESS.2020.3027489.

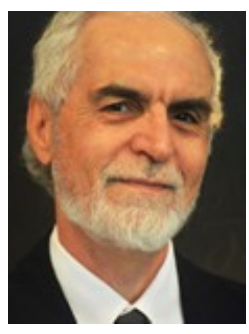

Ivo Barbi (LF'17) was born in Gaspar, Santa Catarina, Brazil. He received the B.S. and M.S. degrees in electrical engineering from the Federal University of Santa Catarina (UFSC), Florianópolis, Brazil, in 1973 and 1976, respectively, and the Dr. Ing. degree in electrical engineering from the Institut National Polytechnique de Toulouse (INPT), Toulouse, France, in 1979.

He founded the Brazilian Power Electronics Society (SOBRAEP) and the Brazilian Power Electronics Conference (COBEP) in 1990, and the Brazilian Power Electronics and Renewable Energy Institute (IBEPE) in 2016. He is a Researcher with the Solar Energy Research Center and a Professor Emeritus in electrical engineering with the Federal University of Santa Catarina.

Prof. Barbi for several years was associate editor of the IEEE Transactions on Industrial Electronics and the IEEE Transactions on Power Electronics. Prof. Barbi received the 2020 IEEE William E. Newell Power Electronics Award. 